\title{
Effect of individual and community-level bed net usage on malaria prevalence among under-fives in the Democratic Republic of Congo
}

\author{
Lauren Levitz ${ }^{1}$, Mark Janko², Kashamuka Mwandagalirwa ${ }^{3}$, Kyaw L. Thwai' ${ }^{1}$ Joris L. Likwela4, \\ Antoinette K. Tshefu ${ }^{3}$, Michael Emch ${ }^{2}$ and Steven R. Meshnick ${ }^{1 *}$
}

\begin{abstract}
Background: Understanding the contribution of community-level long-lasting, insecticidal net (LLIN) coverage to malaria control is critical to planning and assessing intervention campaigns. The Democratic Republic of Congo (DRC), which has one of the highest burdens of malaria cases and deaths and has dramatically scaled up LLIN ownership in recent years thus it is an ideal setting to evaluate the effect of individual versus community-level use to prevent malaria among children under the age of 5 .

Results: Data were derived from the 2013-2014 DRC Demographic and Health Survey. Community-level LLIN usage was significantly associated with protection against malaria, even when individual-level LLIN usage was included in the model. In stratified analysis, higher levels of community LLIN coverage enhanced the protective effect of individual LLIN usage, resulting in lower malaria prevalence among individuals who used a LLIN. A sub-analysis of individual LLIN usage by insecticide type revealed deltamethrin-treated nets were more protective than permethrin-treated nets, suggesting that mosquitoes in the DRC are more susceptible to deltamethrin.

Conclusions: This study examines the effects of individual and community-level LLIN usage in young children in an area of high ITN usage. Individual and community LLIN usage were significantly associated with protection against malaria in children under 5 in the DRC. Importantly, the protective effect of individual LLIN usage against malaria is enhanced when community LLIN coverage is higher, demonstrating the importance of increasing community-level LLIN usage. LLINs treated with deltamethrin were shown to be more protective against malaria than LLINs treated with permethrin. Demographic and Health Surveys are thus a novel and important means of surveillance for insecticide resistance.
\end{abstract}

Keywords: Malaria, Mosquito nets, ITNs, LLINs, DRC, Democratic Republic of Congo, Under-fives, Insecticide resistance

\section{Background}

Malaria causes an estimated 214 million cases and 438,000 deaths yearly, of which the majority occur in children $<5$ years old living in Africa $[1,2]$.

\footnotetext{
*Correspondence: meshnick@unc.edu

${ }^{1}$ Department of Epidemiology, University of North Carolina, Gillings School of Global Public Health, 135 Dauer Drive, 3113 Michael Hooker Research Building, Chapel Hill, NC 27599, USA

Full list of author information is available at the end of the article
}

Insecticide-treated bed nets (ITNs) are a key vector control intervention. ITNs work in three ways: by blocking exposure to potentially infective mosquito bites (preventing transmission from mosquito to human), by preventing contact between a mosquito and a malaria-infected individual (preventing transmission from human to mosquito), and by killing mosquitoes that come into contact with them [3]. The World Health Organization (WHO) estimates ITNs are responsible for preventing $69 \%$ of the 
663 million malaria cases averted due to malaria control interventions between 2001 and 2015 [1]. Since 2007, the WHO has recommended that all ITNs be long-lasting insecticidal nets (LLINs), which are constructed to retain insecticidal activity for at least 20 standard washes under laboratory conditions and 3 years of use in the field [4]. LLINs have been shown to significantly reduce the odds of infection and prevent clinical malaria in children in various settings [5, 6].

While LLINs are an intervention targeted to individuals, coverage of a certain percentage of the population might provide a community effect. Previous studies examining ITN community coverage have shown an association with decreased risk of malaria [7-10]. However, such studies tend to be small, lack generalizability, and often focus on adults or older children. Much of the literature comes from clinical trials or from modelling rather than from research done in the field [1113]. Furthermore, little research has been done in areas of high ITN usage. Understanding the contribution of community-level ITN coverage to malaria risk is critical to planning and assessing intervention campaigns and national control strategies.

The Democratic Republic of Congo (DRC) has one of the highest burdens of malaria cases and deaths, second only to Nigeria [1]. Lack of access to health care, a majority rural population, high poverty, and political instability are contributors to the malaria endemicity [14]. The DRC began distributing ITNs in 2007 [15], and reported ITN ownership increased dramatically by 2013: the percentage of households reporting ownership of at least one net increased from 28 to $72 \%$ for ITNs and from 9 to $70 \%$ for LLINs [16]. By 2014, over a third of all malaria intervention funds (government, Global Fund, President's Malaria Initiative, WHO, United Nations International Children's Emergency Fund, others) went toward ITNs [1]. Given the high malaria burden and current interventional focus on ITNs, the DRC is an ideal setting in which to evaluate the effect of individual versus community-level LLIN usage in preventing malaria.

\section{Methods}

Data were obtained from the second DRC Demographic and Health Survey (DHS) conducted from November 2013 to February 2014 [16]. A total of 536 clusters across all 26 health areas (formerly 11 provinces) were surveyed, comprising 18,360 households. Clustered sampling was designed to allow indicators to be representative at the national level, provincial level, and for urban and rural areas. For 492 of the clusters, global positioning system (GPS) coordinates were recorded using a random displacement method to prevent participant identification. The DHS was unable to collect GPS coordinates from the remaining 44 clusters. Rapid diagnostic tests (RDTs) for malaria and malaria-specific questionnaires were administered to all children ages 6-59 months in half of the study-eligible households.

Dried blood spots (DBS) were collected, stored, and shipped to the University of North Carolina for polymerase-chain reaction (PCR) analysis. DNA was extracted from DBS from 9790 children using Chelex [17], and PCR amplification for Plasmodium falciparum lactate dehydrogenase (DNA) was conducted as described previously [18], with human $\beta$-tubulin as a DNA control [19]. Children who were older than 59 months, were missing GPS coordinates or malaria PCR results, or who were reported to the DHS-DRC II to not be usual residents of the surveyed household, were excluded from further analysis. Since a complete case analysis was performed, children who were missing covariate data were also excluded (Fig. 1).

Data were available for a total of 5857 children; they were included in assessment of multivariate models. Individual- and community-level risk factors were identified a priori based on a review of the literature. Age was defined as the reported age of the child in months. The wealth index was defined by the DHS as economic wellbeing quintiles (lowest, second, middle, fourth, highest), based on self-reported ownership of certain goods (television, radio, car, etc.) and certain household characteristics (including electricity, type of drinking water supply, toilet type, number of sleeping rooms, type of cooking fuel) [16]. Poor household construction is a known risk factor for malaria acquisition [20]; thus a household index was constructed based on survey responses. The household index was coded from 0 to 6 , with two points assigned for "finished," one for "rudimentary," and zero for "natural" for each of the three housing categories (roof, floor, walls). Mother's highest education level was coded from 0 to 3, corresponding to no education, primary school, secondary school, or higher education. Regional-level 2007 malaria prevalence was calculated by plotting the cluster GPS coordinates onto the 2007 P. falciparum malaria prevalence map previously [21]. Malaria prevalence was dichotomized as low $(<50 \%)$ or high $(\geq 50 \%)$. This variable was assessed for inclusion because malaria is persistent in space over time, and historical malaria prevalence might influence current LLIN usage. Community LLIN usage was calculated as the percentage of children within a given cluster who reported using a LLIN the previous night divided by the total number of observations in that cluster. Individual LLIN usage the previous night was coded as a dichotomous yes/no for LLIN usage the previous night. Children who reported using an untreated net were coded as not having used a LLIN the night before. 


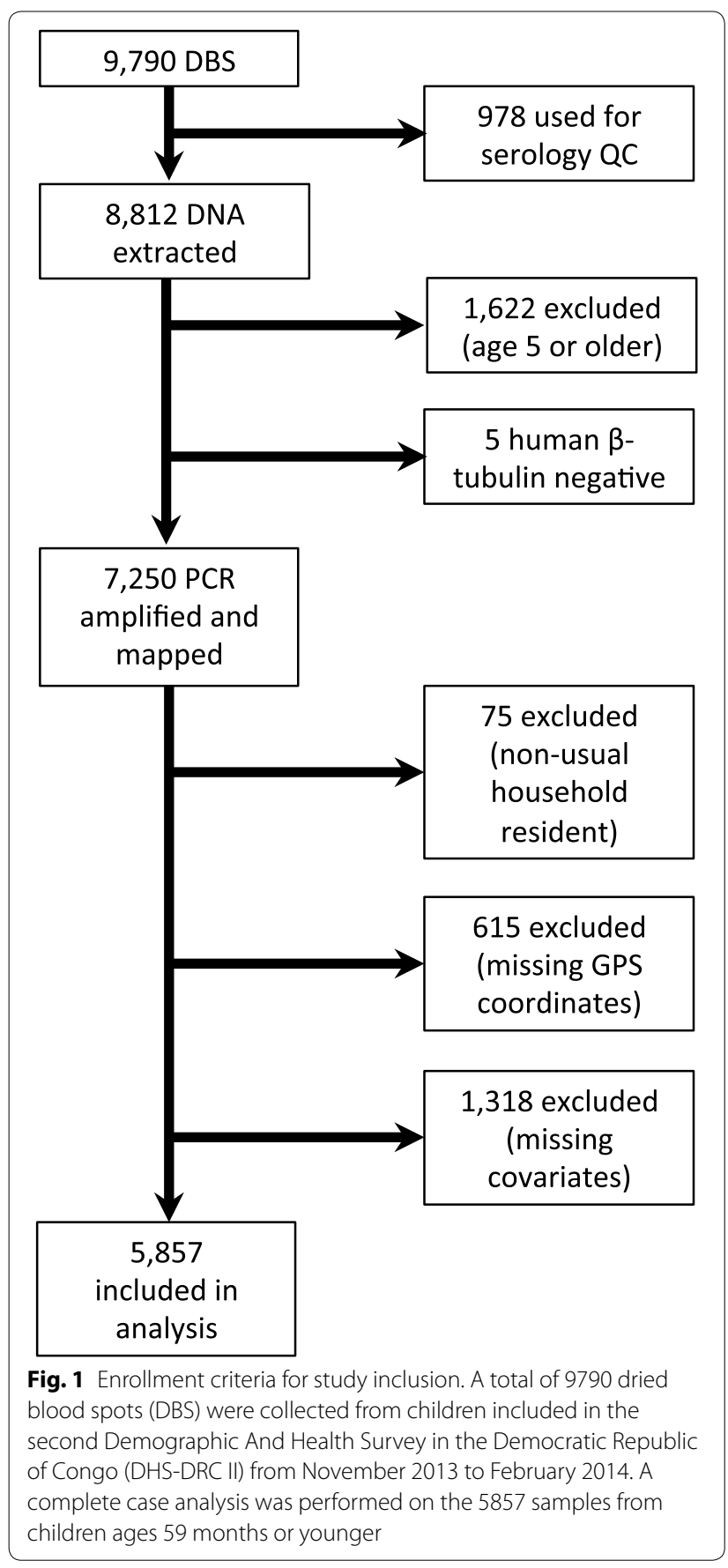

In order to assess both individual and community level variables, multilevel models were constructed. Multilevel models adjust for the collinearity of individual and cluster-level variables. Three crude multilevel logistic regression models were constructed to test association with malaria infection: one with individual LLIN usage (Model 1), one with community LLIN usage (Model 2), and one with both (full model). The outcome variable was a dichotomous positive/negative malaria diagnosis for P. falciparum as assessed by real-time PCR. The models were built in SAS v. 9.4 (SAS Institute, Cary, NC) using PROC SURVEYLOGISTIC adjusted for DHS-DRC II sampling weights. A multilevel modelling approach is necessary to take into account the correlation of individuals within clusters. Expected relationships between risk factors and malaria diagnosis were defined a priori. Non-significant variables were removed from the model by backwards selection. The three models were assessed by Akaike's Information Criterion (AIC), which penalizes over-fitting and favours parsimony, with lower AIC values preferred. Model 1 included the following variables: age in months, housing index, mother's highest educational level, individual LLIN usage, and cluster altitude in meters. Model 2 included the following variables: age in months, housing index, mother's highest educational level, cluster LLIN usage, and cluster altitude in meters. Model 3 included the following variables: age in months, housing index, mother's highest educational level, individual LLIN usage, cluster LLIN usage, and cluster altitude in meters.

It is plausible that individuals who did not sleep under a LLIN might benefit if there is a protective effect of community LLIN usage. While previous studies have demonstrated a positive association between community LLIN usage $\geq 50 \%$ and decreased malaria infection among children in households possessing at least one ITN [22, 23], there is not a definitive threshold that is protective. Thus to determine whether the effect of individual LLIN usage is modified by varying levels of communitylevel LLIN usage, the community-level LLIN usage variable was stratified into quartiles. Three interaction terms between the community-level coverage variables and individual-level LLIN usage was included to test for the presence of effect modification of the odds ratio. Homogeneity of the stratum-specific exposure odds ratio estimates was assessed by likelihood ratio test (LRT) at an a priori level of 0.05 .

For those who reported using a LLIN the previous night, LLIN brand was also reported. LLIN brands were recoded by insecticide type as follows: deltamethrin (PermaNet, Yorkool LN, Lifenet, Serena, Netprotect); permethrin (Olyset); alpha-cypermethrin (Duranet, Magnet, Interceptor); and brand not specified. Model 1 was modified to test the association between individual use of a LLIN treated with a particular insecticide (deltamethrin, permethrin, alpha-cypermethrin, or brand not specified) and odds of PCR-positive malaria; the four insecticides were tested in four separate models, with no LLIN usage as the referent group. 


\section{Results}

Of the 5857 individuals included in this study, 37.4\% were PCR-positive for P. falciparum and $53.6 \%$ had slept under a bed night the previous night. A summary of the prevalences of malaria by covariate is presented in Table 1 . Factors which were significantly associated with malaria prevalence include age, housing quality, wealth, mother's education, individual and community of bednets, prevalence in 2007, rurality, type of bed net insecticide, and altitude. No significant differences were seen by gender or bednet age.

Six multilevel models were considered: three crude models (full model including both individual- and community-level LLIN usage, Model 1 with only individual-level LLIN usage, and Model 2 with only community-level LLIN usage) and three adjusted models. Only variables that were significant at an alpha level of $\mathrm{p}<0.05$ were included in a given adjusted model. Covariates that were significant in all three models include age in months, housing index, mother's highest educational level, and cluster altitude.

Prevalence odds ratios (OR) for the three models are presented in Table 2 . The crude models include only individual-level LLIN usage, community-level LLIN usage, or both. The adjusted models include covariates identified as significant as described in Methods. In both Model 1 and Model 3, individual LLIN usage resulted in a significant decrease in the OR of PCR-positive malaria. This held true after adjustment for covariates. In Models 2 and 3, community-level LLIN usage significantly reduced the odds of PCR-positive malaria after adjustment for covariates, but not in the crude models. The best fitting model as determined by the lowest AIC was Model 3 adjusted for covariates (AIC 7127.47), which includes both individual-(OR $0.77,95 \%$ CI $0.66,0.91)$ and communitylevel LLIN usage (OR 0.56, 95\% CI 0.33, 0.95).

To determine whether the effect of individual LLIN usage is modified by varying levels of community-level LLIN usage, the community-level coverage variable was stratified into quartiles. This was coded as three disjoint indicator variables and added to the final model (Model 3 , adjusted). Stratum-specific odds ratio estimates are presented in Table 3. There is a trend toward an increased protective effect of individual LLIN usage as communitylevel LLIN usage increases. Individual LLIN usage is most protective against PCR-positive malaria at the highest quartile of community-level LLIN usage (OR 0.47, 95\% CI $0.30,0.73)$. The test for homogeneity was significant, indicating there is a departure from perfect multiplicativity of the odds ratio, but there is a high degree of overlap of the $95 \%$ confidence intervals for the stratum-specific estimates. Notably, higher community-level LLIN usage led to a decrease in malaria prevalence, but only among
Table 1 Prevalence of PCR-positive malaria among subjects

\begin{tabular}{|c|c|c|}
\hline Variable (n) & $\%$ PCR positive & $p$ value $*$ \\
\hline \multicolumn{3}{|l|}{ Age } \\
\hline < 1 year (658) & 29 & \multirow[t]{5}{*}{$<0.001$} \\
\hline $1-2$ years (1388) & 31 & \\
\hline $2-3$ years (1303) & 38 & \\
\hline $3-4$ years (1312) & 42 & \\
\hline 4-5 years (1189) & 43 & \\
\hline \multicolumn{3}{|l|}{ Sex } \\
\hline Male (2935) & 38 & \multirow[t]{2}{*}{0.365} \\
\hline Female (2915) & 37 & \\
\hline \multicolumn{3}{|l|}{ Housing quality index } \\
\hline Lowest quality (786) & 31 & \multirow[t]{7}{*}{$<0.001$} \\
\hline 2nd level (3118) & 42 & \\
\hline 3rd level (365) & 40 & \\
\hline 4th level (403) & 35 & \\
\hline 5th level (459) & 34 & \\
\hline 6th level (71) & 25 & \\
\hline Highest quality (648) & 23 & \\
\hline \multicolumn{3}{|l|}{ Wealth index } \\
\hline Lowest quintile (1558) & 40 & \multirow[t]{5}{*}{$<0.001$} \\
\hline 2nd quintile (1366) & 40 & \\
\hline Middle quintile (1153) & 39 & \\
\hline 4th quintile (1034) & 39 & \\
\hline Highest quintile (739) & 22 & \\
\hline \multicolumn{3}{|l|}{ Mother's education } \\
\hline None (1255) & 41 & \multirow[t]{4}{*}{$<0.001$} \\
\hline Primary (2633) & 41 & \\
\hline Secondary (1903) & 30 & \\
\hline Higher (59) & 19 & \\
\hline \multicolumn{3}{|c|}{ Number of household members } \\
\hline $2-4(1191)$ & 36 & \multirow[t]{4}{*}{0.505} \\
\hline $5-7(2629)$ & 37 & \\
\hline $8-10(1510)$ & 39 & \\
\hline$>10(520)$ & 36 & \\
\hline \multicolumn{3}{|c|}{ Respondent slept under LLIN previous night } \\
\hline Yes (2700) & 42 & \multirow[t]{2}{*}{$<0.001$} \\
\hline No $(3150)$ & 34 & \\
\hline \multicolumn{3}{|l|}{ Altitude (m) } \\
\hline$<500(2165)$ & 38 & \multirow[t]{4}{*}{$<0.001$} \\
\hline $500-1000(2609)$ & 42 & \\
\hline $1000-1500$ (629) & 35 & \\
\hline $1500-2000(447)$ & 9 & \\
\hline \multicolumn{3}{|l|}{2007 prevalence } \\
\hline High (2783) & 44 & \multirow[t]{2}{*}{$<0.001$} \\
\hline Low (3067) & 32 & \\
\hline \multicolumn{3}{|c|}{ \% Cluster LLIN coverage previous night (quartiles) } \\
\hline $0-33(1202)$ & 42 & \multirow[t]{4}{*}{$<0.001$} \\
\hline $34-54(1688)$ & 40 & \\
\hline $55-75(1495)$ & 36 & \\
\hline $76-100(1321)$ & 31 & \\
\hline
\end{tabular}


Table 1 continued

\begin{tabular}{llc}
\hline Variable $(\mathbf{n})$ & \% PCR positive & $\boldsymbol{p}$ value* \\
\hline Time to water source (min) & 37 & \\
$0-12(1222)$ & 40 & \\
$12-30(2304)$ & 35 & \\
$30-45(662)$ & 37 & \\
Over 45 (1400) & & \\
Urban/rural residence & 35 & \\
Urban (1772) & 38 & \\
Rural (4078) & & \\
Bed net age & 34 & \\
$<1$ year (824) & 31 & \\
1-2 years (1440) & 36 & \\
2-3 years (479) & 38 & \\
$>3$ years (475) & & \\
Insecticide & 24 & \\
Alphacypermethrin (59) & 32 & \\
Deltamethrin (2592) & 42 & \\
Permethrin (445) &
\end{tabular}

* Chi square test for null hypothesis that all prevalences are equal

those who used a LLIN themselves, indicating the importance of individual LLIN usage.

Prevalence odds ratio estimates for individual LLIN usage by insecticide type are shown in Fig. 2a (with raw data in Fig. 2b). Use of a deltamethrin-treated net significantly reduced the odds of PCR-positive malaria as compared to no LLIN use in both crude (OR 0.70, 95\% CI 0.57, 0.85 ) and adjusted (OR 0.62, 95\% CI 0.51, 0.75) models. Alpha-cypermethrin-treated nets led to even lower ORs, but these were not significant in the crude (OR 0.39, 95\% CI $0.14,1.10$ ) or adjusted (OR $0.53,95 \%$ CI $0.21,1.35$ ) models, likely due to very small sample size. Permethrintreated nets led to non-significant ORs close to the null in both crude (OR 0.91, 95\% CI 0.51, 1.63) and adjusted (OR $0.89,95 \%$ CI $0.55,1.44)$ models. Malaria prevalence among those who used deltamethrin-treated nets was $32.2 \%$, as compared to $42.1 \%$ among those who used permethrintreated nets and $41.9 \%$ among those who did not use a LLIN (Fig. 2b). The observed differences were not due to bed net age; the median reported ages (IQR) of the bednets were 13 (11-24) months, 16 (12-24) months and 13 (12-24) months, for deltamethrin-, alpha-cypermethrinand permethrin-treated nets, respectively, and were not significantly different.

\section{Discussion}

These results demonstrate the importance of both individual- and community-level LLIN usage in preventing malaria among those most at risk of infection in subSaharan African, children under the age of 5. Furthermore, this study examines the effects of individual as well as community-level LLIN usage in young children in an area of high ITN usage. High LLIN usage was significantly associated with protection against malaria, even when individual-level LLIN usage was included in the model. Importantly, higher levels of community LLIN coverage enhanced the protective effect of individual LLIN usage, resulting in lower malaria prevalence among individuals who used a LLIN. Deltamethrin-treated nets were more protective than permethrin-treated nets. However, since the nets were not randomly distributed, the observed differences could have been affected by selection bias.

The significant effect of individual-level LLIN usage on odds of malaria is different from what was observed in the DRC in 2007, where ITN usage was found to be protective at the community level but not at the individual level [9]. This could be due to several reasons. First, the 2007 DHS-DRC survey included adults ages 15-59 and excluded children [9] whereas the current study population is exclusively children. Adults are more likely to be outside during peak-biting evening hours [24-26], potentially increasing their exposure to malarial mosquitoes. Consequently, community-level ITN coverage might be more important for malaria prevention at older ages. Second, the distribution of treated versus untreated nets in the DRC has shifted substantially since the 2007 DHSDRC: while roughly twice as many survey respondents reported sleeping under an untreated net as compared to a treated net in the 2007 DHS-DRC [9], the vast majority of DHS-DRC II respondents slept under a treated net

Table 2 Comparison of odds ratios and model fit across the three models

\begin{tabular}{|c|c|c|c|c|c|c|}
\hline & \multicolumn{2}{|c|}{ Model 1: individual LLIN use } & \multicolumn{2}{|c|}{ Model 2: community LLIN use } & \multicolumn{2}{|l|}{ Model 3: both } \\
\hline & Crude & Adjusted & Crude & Adjusted & Crude & Adjusted \\
\hline \multicolumn{7}{|l|}{ Odds ratio $(95 \% \mathrm{Cl})$} \\
\hline Individual LLIN use & $0.72(0.61,0.87)$ & $0.65(0.55,0.77)$ & & & $0.75(0.64,0.88)$ & $0.77(0.66,0.91)$ \\
\hline Community LLIN use & & & $0.67(0.40,1.12)$ & $0.43(0.27,0.70)$ & $0.89(0.51,1.55)$ & $0.56(0.33,0.95)$ \\
\hline$A / C$ & 7438.04 & 7127.89 & 7455.49 & 7121.07 & 7439.11 & 7109.15 \\
\hline
\end{tabular}


Table 3 Effect of community LLIN use on the protective effect of individual LLIN use

\begin{tabular}{|c|c|c|c|c|c|c|}
\hline \multirow[t]{2}{*}{ Quartile of community-level LLIN usage } & \multirow[t]{2}{*}{ Individual LLIN OR (95\% Cl) } & \multicolumn{2}{|c|}{ Malaria prevalence (\%) } & \multirow[t]{2}{*}{ LRT statistic } & \multirow[t]{2}{*}{ DF } & \multirow[t]{2}{*}{$p$ value } \\
\hline & & Individual LLIN & No individual LLIN & & & \\
\hline Lowest quartile & $0.81(0.58,1.15)$ & 39.4 & 42.4 & 12.37 & 3 & 0.01 \\
\hline Second quartile & $0.87(0.68,1.11)$ & 38.5 & 42.7 & & & \\
\hline Third quartile & $0.71(0.53,0.95)$ & 34.1 & 39.4 & & & \\
\hline Highest quartile & $0.47(0.31,0.69)$ & 29.2 & 42.3 & & & \\
\hline
\end{tabular}

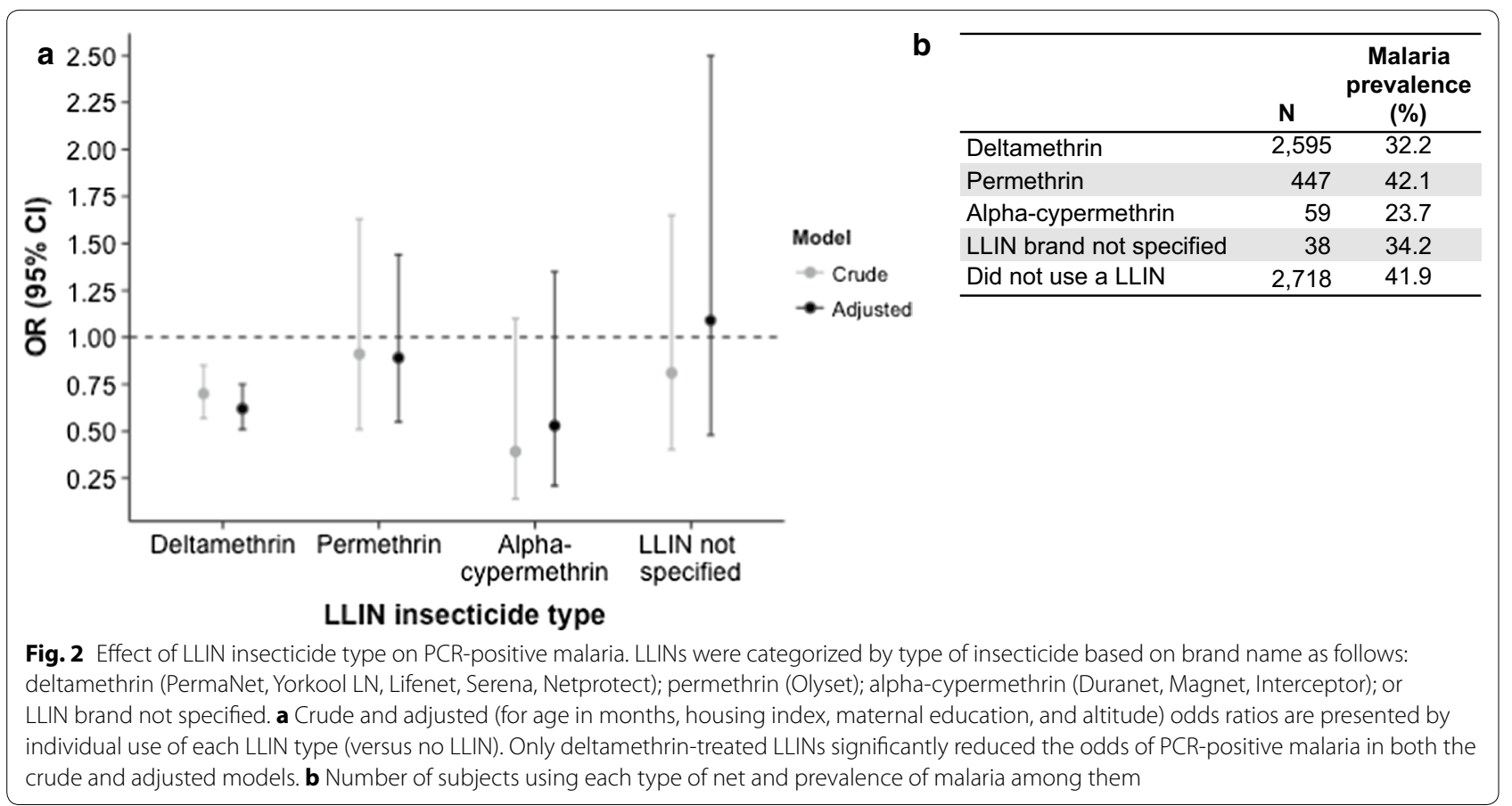

[16]. This dramatic increase in ITNs and LLINs from 2007 to 2013-2014 could lead to a larger individual protective effect and reduce the relative importance of community coverage. The effect of individual LLIN usage is modified by community-level LLIN usage, and stratified analysis indicates that individual net usage is more protective when community coverage is high.

Despite mass distribution campaigns, LLIN usage remains below full coverage of the at-risk population. Factors such as household wealth, age, educational status of the head of the household, number of children underfive, malaria endemicity, and distance to health centers have been demonstrated to affect children's ITN usage in other African settings [27]. Education campaigns have been shown to significantly increase ITN usage [28, 29], and cost of ITN (free versus subsidized versus full market price) influences ITN ownership though likely not ITN usage [29].
The 2013-2014 DHS-DRC did not obtain information about why survey respondents did or did not choose to use a LLIN. Based on results from the full 2013-14 DHSDRC, overall access to an ITN in the DRC was found to be $47 \%$, with access dependent on several factors, including province (ranging from $62.6 \%$ in Bandundu to $31 \%$ in Kasai-Occidental) and number of people sleeping in the household (ranging from $63.7 \%$ for 2 people to $38.1 \%$ for 8 people) [16]. In order to make policy recommendations about future ITN campaigns, more research is needed to determine geographic areas to prioritize and methods to improve ITN usage in this population following distributions. This would allow for the development of highly targeted ITN campaigns paired with culturally appropriate education campaigns.

Insecticide resistance is a growing concern, and pyrethroid resistance among Anopheles mosquitoes has been reported in the DRC [30-32]. The three most commonly 
used LLIN brands reported in the 2013-14 DHS-DRC are treated with deltamethrin (PermaNet, Serena) or permethrin (Olyset). A field efficacy trial in Kinshasa demonstrated significantly higher bioefficacy of PermaNet 3.0 LLINs against Anopheles gambiae mosquitoes from the DRC as compared to OlysetNet; furthermore, individuals sleeping under PermaNet 3.0 LLINs had lower biting rates and reported better sleep quality as compared to those sleeping under OlysetNet [32]. The authors note, however, that the improved effectiveness of PermaNet 3.0 might be attributed to both the deltamethrin and the addition of piperonyl butoxide (PBO), which increases insecticide penetration and inhibits metabolic detoxification [32]. Given these results, it is encouraging that the vast majority of LLINs used by children in the current study were PermaNet or other deltamethrin-treated brands. As examination of insecticide resistance or bioefficacy of LLINs was outside the scope of the current project, the observed increased protection of deltamethrin-treated nets might also be attributed to additional factors such as age and condition of the net or distribution of permethrin-treated nets to higher-transmission areas. Phenotypic resistance to all three pyrethroids has been documented in field-collected A. gambiae mosquitoes from the DRC [31]. If there is a high degree of insecticide resistance in the study area, the effect of community-level LLIN usage on the odds of PCR-positive malaria might be diminished due to reduced killing of infectious mosquitoes that come into contact with treated nets. Consequently, individual use of a bed net might become more important, as the nets would act as a barrier to prevent exposure even if the insecticides are less effective. This is supported by the increased effect size of individual LLIN usage on the odds of PCR-positive malaria among communities with higher as compared to lower levels of LLIN coverage. Examination of insecticide resistance is an important area for future research.

This study was limited by the cross-sectional design of the DHS-DRC. Cross-sectional studies are useful in identifying associations but are unable to establish causality. Malaria prevalence (and likely ITN usage) varies seasonally, meaning that the effect of individual versus community-level ITN usage on malaria risk might depend on season as well. A longitudinal study in the Kinshasa province is currently underway, which will be able to address such questions.

\section{Conclusions}

Individual and community usage of LLINs is significantly associated with protection against malaria in children under the age of 5 . Importantly, the protective effect of individual LLIN usage against malaria is enhanced when community LLIN coverage is higher, demonstrating the importance of increasing community-level LLIN usage. LLINs treated with deltamethrin were shown to be more protective against malaria than LLINs treated with permethrin. Thus, DHS surveys are a rich potential sources of information about insecticide resistance.

\begin{abstract}
Abbreviations
DBS: dried blood spot; DHS: Demographic and Health Survey; DRC: Democratic Republic of the Congo; ITN: insecticide-treated net; LLIN: long-lasting, insecticidal net; PCR: polymerase-chain reaction; RDT: rapid diagnostic test; WHO: World Health Organization.
\end{abstract}

\section{Authors' contributions}

LL analysed the data and drafted the manuscript. MJ provided statistical advice and helped to draft the manuscript. KLT carried out the PCR analyses. KW, JLL, and AKT provided study coordination from the DRC. ME and SRM conceived of and coordinated the study. All authors consented to its publication. All authors read and approved the final manuscript.

\section{Author details}

${ }^{1}$ Department of Epidemiology, University of North Carolina, Gillings School of Global Public Health, 135 Dauer Drive, 3113 Michael Hooker Research Building, Chapel Hill, NC 27599, USA. ${ }^{2}$ Department of Geography, University of North Carolina Gillings School of Global Public Health, Chapel Hill, NC 27599, USA. ${ }^{3}$ University of Kinshasa School of Public Health, Kinshasa, Democratic Republic of Congo. ${ }^{4}$ Programme National de la Lutte contre le Paludisme, Kinshasa, Democratic Republic of Congo.

\section{Acknowledgements}

We are deeply grateful to the organizers of and participants in the 2013-2014 DHS in the DRC. This study would not have been achievable without their hard work and participation. We would also like to acknowledge Stephanie Doctor and Jonathan Parr for their input and technical assistance.

\section{Competing interests}

The authors declare that they have no competing interests.

\section{Availability of data and materials}

Sharing of individual-level raw PCR data is not permitted under current Institutional Review Board approvals. The 2013-2014 DHS-DRC is accessible on the DHS website after registration: http://dhsprogram.com/data/dataset/CongoDemocratic-Republic_Standard-DHS_2013.cfm.

\section{Ethics approval and consent to participate}

Parental consent for children's participation in the 2013-2014 Demographic and Health Surveys (DHS) was obtained by the DHS Program. The 2013-2014 DRC DHS was reviewed and approved by the ICF International Institutional Review Board (IRB) and the University of Kinshasa IRB (Comité d'Ethique de I'Ecole de Santé Publique de l'Université de Kinshasa). This study was approved by the University of North Carolina IRB.

\section{Funding}

This study was supported by the National Institutes of Health Grant R01Al107949 to SRM. MJ received support from the Population Research Infrastructure Program through funding awarded by the Eunice Kennedy Shriver National Institute of Child Health and Human Development to the Carolina Population Center [P2C HD050924]. ME received support from the National Science Foundation Grant BCS-1339949. The funders had no role in study design, data collection and interpretation, or the decision to submit the work for publication.

\section{Publisher's Note}

Springer Nature remains neutral with regard to jurisdictional claims in published maps and institutional affiliations. 
Received: 19 April 2017 Accepted: 10 January 2018

Published online: 18 January 2018

\section{References}

1. WHO. World malaria report 2015. Geneva: World Health Organization; 2015. http://apps.who.int/iris/bitstream/10665/200018/1/9789241565158_eng. pdf?ua=1. Accessed 22 Aug 2016.

2. Laishram DD, Sutton PL, Nanda N, Sharma VL, Sobti RC, Carlton JM, et al. The complexities of malaria disease manifestations with a focus on asymptomatic malaria. Malar J. 2012;11:29.

3. Eisele TP, Steketee RW. African malaria control programs deliver ITNs and achieve what the clinical trials predicted. PLoS Med. 2011;8:e1001088.

4. WHO. Guidelines for laboratory and field testing of long-lasting insecticidal nets. Geneva: World Health Organization; 2013. http://apps.who. int/iris/bitstream/10665/80270/1/9789241505277_eng.pdf. Accessed 19 Sept 2017.

5. Lengeler $C$. Insecticide-treated bed nets and curtains for preventing malaria. Cochrane Database Syst Rev. 2004;2:CD00036.

6. Damien GB, Djènontin A, Chaffa E, Yamadjako S, Drame PM, Ndille EE, et al. Effectiveness of insecticidal nets on uncomplicated clinical malaria: a case-control study for operational evaluation. Malar J. 2016;15:102.

7. Plucinski MM, Chicuecue S, Macete E, Colborn J, Yoon SS, Kachur SP, et al. Evaluation of a universal coverage bed net distribution campaign in four districts in Sofala Province, Mozambique. Malar J. 2014;13:427.

8. Kesteman T, Randrianarivelojosia M, Piola P, Rogier C. Post-deployment effectiveness of malaria control interventions on Plasmodium infections in Madagascar: a comprehensive phase IV assessment. Malaria J. 2016;15:322.

9. Messina JP, Taylor SM, Meshnick SR, Linke AM, Tshefu AK, Atua B, et al. Population, behavioural and environmental drivers of malaria prevalence in the Democratic Republic of Congo. Malar J. 2011;10:161.

10. Mathanga DP, Mwandama DA, Bauleni A, Chisaka J, Shah MP, Landman $K Z$, et al. The effectiveness of long-lasting, insecticide-treated nets in a setting of pyrethroid resistance: a case-control study among febrile children 6 to 59 months of age in Machinga District, Malawi. Malar J. 2015;14:457.

11. Binka FN, Indome F, Smith T. Impact of spatial distribution of permethrinimpregnated bed nets on child mortality in rural northern Ghana. Am J Trop Med Hyg. 1998;59:80-5.

12. Hawley WA, Phillips-Howard PA, ter Kuile OF, Terlouw DJ, Vulule JM, Ombok M, et al. Community-wide effects of permethrin-treated bed nets on child mortality and malaria morbidity in western Kenya. Am J Trop Med Hyg. 2003;68(4 Suppl):121-7.

13. Maxwell CA, Msuya E, Sudi M, Njunwa K, Carneiro I, Curtis C. Effect of community-wide use of insecticide-treated nets for 3-4 years on malarial morbidity in Tanzania. Trop Med Int Health. 2002;7:1003-8.

14. Benedetti G, Mossoko M, Kakusu JPN, Nyembo J, Mangion JP, Van Laeken D, et al. Sparks creating light? Strengthening peripheral disease surveillance in the Democratic Republic of Congo. Public Health Action. 2016:6:54-9.

15. Ferrari G, Ntuku HM, Schmidlin S, Diboulo E, Tshefu AK, Lengeler C. A malaria risk map of Kinshasa, Democratic Republic of Congo. Malar J. 2016;15:27.

16. Ministère du Plan et Suivi de la Mise en œuvre de la Révolution de la Modernité (MPSMRM), Ministère de la Santé Publique (MSP), ICF International. Enquête Démographique et de Santé en République Démocratique du Congo 2013-2014. Rockville: MPSMRM, MSP and ICF International; 2014
17. Okell LC, Ghani AC, Lyons E, Drakeley CJ. Submicroscopic infection in Plasmodium falciparum-endemic populations: a systematic review and meta-analysis. J Infect Dis. 2009:200:1509-17.

18. Singh B, Bobogare A, Cox-Singh J, Snounou G, Abdullah MS, Rahman HA. A genus- and species-specific nested polymerase chain reaction malaria detection assay for epidemiologic studies. Am J Trop Med Hyg. 1999;60:687-92.

19. Beshir KB, Hallett RL, Eziefula AC, Bailey R, Watson J, Wright SG, et al. Measuring the efficacy of anti-malarial drugs in vivo: quantitative PCR measurement of parasite clearance. Malar J. 2010;9:312.

20. Snyman K, Mwangwa F, Bigira V, Kapisi J, Clark TD, Osterbauer B, et al. Poor housing construction associated with increased malaria incidence in a cohort of young Ugandan children. Am J Trop Med Hyg. 2015:92:1207-13.

21. Taylor SM, Messina JP, Hand CC, Juliano JJ, Muwonga J, Tshefu AK, et al. Molecular malaria epidemiology: mapping and burden estimates for the Democratic Republic of the Congo, 2007. PLoS ONE. 2011;6:e16420.

22. Larsen DA, Hutchinson P, Bennett A, Yukich J, Anglewicz P, Keating J, et al. Community coverage with insecticide-treated mosquito nets and observed associations with all-cause child mortality and malaria parasite infections. Am J Trop Med Hyg. 2014;91:950-8.

23. Killeen GF, Smith TA, Ferguson HM, Mshinda H, Abdulla S, Lengeler C, et al. Preventing childhood malaria in Africa by protecting adults from mosquitoes with insecticide-treated nets. PLoS Med. 2007;4:e229.

24. Cooke MK, Kahindi SC, Oriango RM, Owaga C, Ayoma E, Mabuka D, et al. 'A bite before bed': exposure to malaria vectors outside the times of net use in the highlands of western Kenya. Malar J. 2015;14:259.

25. Shililu J, Ghebremeskel T, Seulu F, Mengistu S, Fekadu H, Zerom M, et al. Seasonal abundance, vector behavior, and malaria parasite transmission in Eritrea. JAMA. 2004;20:155-64.

26. Fornadel CM, Norris LC, Glass GE, Norris DE. Analysis of Anopheles arabiensis blood feeding behavior in southern Zambia during the 2 years after introduction of insecticide-treated bed nets. Am J Trop Med Hyg. 2010;83:848-53.

27. Njau JD, Stephenson R, Menon M, Kachur SP, McFarland DA. Exploring the impact of targeted distribution of free bed nets on households bed net ownership, socio-economic disparities and childhood malaria infection rates: analysis of national malaria survey data from three sub-Saharan Africa countries. Malar J. 2013;12:245.

28. Rhee M, Sissoko M, Perry S, McFarland W, Parsonnet J, Doumbo O. Use of insecticide-treated nets (ITNs) following a malaria education intervention in Piron, Mali: a control trial with systematic allocation of households. Malar J. 2005;4:35

29. Augustincic Polec L, Petkovic J, Welch V, Ueffing E, Ghogomu ET, Pardo $J$, et al. Strategies to increase the ownership and use of insecticidetreated bednets to prevent malaria. Cochrane Database Syst Rev. 2015;3:CD009186.

30. Knox TB, Juma EO, Ochomo EO, Pates Jamet H, Ndungo L, Chege P, et al. An online tool for mapping insecticide resistance in major Anopheles vectors of human malaria parasites and review of resistance status for the Afrotropical region. Parasit Vectors. 2014;7:76.

31. Basilua Kanza JP, El Fahime E, Alaoui S, el Essassi M, Brooke B, Nkebolo Malafu A, et al. Pyrethroid, DDT and malathion resistance in the malaria vector Anopheles gambiae from the Democratic Republic of Congo. Trans R Soc Trop Med Hyg. 2013:107:8-14.

32. Bobanga T, Ayieko W, Zanga M, Umesumbu S, Landela A, Fataki O, et al. Field efficacy and acceptability of PermaNet ${ }^{\circledR} 3.0$ and OlysetNet ${ }^{\circledR}$ in Kinshasa, Democratic Republic of the Congo. J Vector Borne Dis. 2013:50:206-14. 